\title{
Hypergeometric formulas for lattice sums and Mahler measures
}

\author{
Mathew Rogers* \\ Department of Mathematics, University of Illinois \\ Urbana, IL 61801, USA
}

October 23, 2018

\begin{abstract}
In this paper, we will prove several formulas relating generalized hypergeometric functions to lattice sums with four indices of summation. These results are related to Boyd's conjectured identities between Mahler measures and special values of $L$-series of elliptic curves.
\end{abstract}

\section{Introduction}

In this paper we will prove formulas relating lattice sums to hypergeometric functions. This research was inspired by the work of Boyd, who used numerical methods to conjecture hundreds of relations between the $L$-series of elliptic curves and special values of Mahler's measure [7]. The first example of such an identity was due to Deninger [11, who hypothesized that

$$
\mathrm{m}\left(1+y+y^{-1}+z+z^{-1}\right) \stackrel{?}{=} \frac{15}{4 \pi^{2}} L(E, 2)
$$

where $E$ is a conductor 15 elliptic curve. As usual, "? " denotes a conjectured equality which holds to at least 50 decimal places. The Mahler measure of a $n$ dimensional polynomial is defined in equation (2.2). Boyd observed that since every

${ }^{*}$ The author is supported by NSF award DMS-0803107 
elliptic curve is modular, this identity can be translated into a completely explicit formula:

$$
\int_{0}^{1} \int_{0}^{1} \log |1+2 \cos (2 \pi t)+2 \cos (2 \pi s)| \mathrm{d} s \mathrm{~d} t \stackrel{?}{=} \frac{15}{4 \pi^{2}} \sum_{n=1}^{\infty} \frac{a_{n}}{n^{2}}
$$

where

$$
\sum_{n=1}^{\infty} a_{n} q^{n}=q \prod_{n=1}^{\infty}\left(1-q^{n}\right)\left(1-q^{3 n}\right)\left(1-q^{5 n}\right)\left(1-q^{15 n}\right)
$$

If this line of thought is pushed somewhat further (see Theorem 2.1), the following conjecture arises:

$$
\begin{aligned}
\sum_{n=0}^{\infty}\left(\begin{array}{c}
2 n \\
n
\end{array}\right)^{2} \frac{(1 / 16)^{2 n+1}}{2 n+1} \\
\quad \stackrel{?}{=} \frac{540}{\pi^{2}} \sum_{n_{i}=-\infty}^{\infty} \frac{(-1)^{n_{1}+n_{2}+n_{3}+n_{4}}}{\left(\left(6 n_{1}+1\right)^{2}+3\left(6 n_{2}+1\right)^{2}+5\left(6 n_{3}+1\right)^{2}+15\left(6 n_{4}+1\right)^{2}\right)^{2}} .
\end{aligned}
$$

Equation (1.3) is an interesting conjecture, because it relates a complicated lattice sum to the ${ }_{3} F_{2}$ hypergeometric function. Lattice sums have been extensively studied in physics, since they often arise when calculating electrostatic potentials of crystal lattices (for instance see [15], 25] and [14]). It is often difficult to calculate lattice sums numerically, and it is quite unusual to be able to reduce them to known special functions [6]. The problem of finding a closed form for Madelung's constant is probably the most famous open problem in this area [10].

Definition 1.1. Let us define $F(a, b, c, d)$ by

$$
\begin{aligned}
F(a, b, c, d):= & (a+b+c+d)^{2} \\
& \times \sum_{n_{i}=-\infty}^{\infty} \frac{(-1)^{n_{1}+n_{2}+n_{3}+n_{4}}}{\left(a\left(6 n_{1}+1\right)^{2}+b\left(6 n_{2}+1\right)^{2}+c\left(6 n_{3}+1\right)^{2}+d\left(6 n_{4}+1\right)^{2}\right)^{2}} .
\end{aligned}
$$

We will also fix the following shorthand notation:

$$
F(b, c):=F(1, b, c, b c) .
$$

Finally, the default method of summation will be $\sum_{n_{i}=-\infty}^{\infty}=\lim _{v \rightarrow \infty} \sum_{n_{1}=-v}^{v} \cdots \sum_{n_{4}=-v}^{v}$.

In this paper, we will prove many new formulas for special values of $F(a, b, c, d)$. While we believe that it should be possible to find a general formula for the function, we have not managed to accomplish that goal yet. The second and third sections of the paper summarize 18 formulas relating $F(b, c)$ to rational hypergeometric functions. Virtually all of those identities were extracted directly from Boyd's tables 
7]. For example, Boyd conjectured a formula for a conductor 20 elliptic curve, which is equivalent to:

$$
\frac{25}{6 \pi^{2}} F(1,5) \stackrel{?}{=} \sqrt[3]{2} A_{3} F_{2}\left(\begin{array}{c}
\frac{1}{3}, \frac{1}{3}, \frac{1}{3}, \frac{2}{3}, \frac{4}{3} ; \\
27
\end{array}\right)+\sqrt[3]{4} B_{3} F_{2}\left(\begin{array}{c}
\frac{2}{3}, \frac{2}{3}, \frac{2}{3}, \\
\frac{4}{3}, \frac{5}{3} ; \frac{2}{27}
\end{array}\right)
$$

where $A$ and $B$ are given in terms of gamma functions (see Theorem [3.1). When more general lattice sums are considered, hypergeometric functions with irrational arguments frequently appear. For instance, if $\phi=\frac{1+\sqrt{5}}{2}$, we have

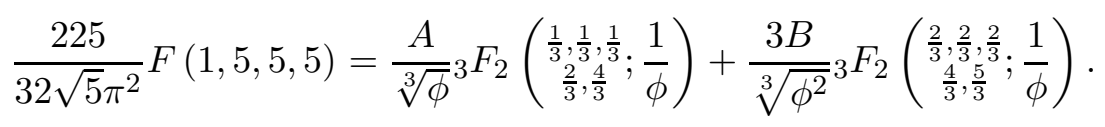

Formula (1.6) closely resembles identities that Forrester and Glasser established for three-dimensional sums associated with $\mathrm{NaCl}$ lattices [14]. While it seems likely that equations (1.6) and (1.5) both arise as special cases of formulas for $F(a, b, c, d)$, the exact nature of those formulas remains unclear. From our calculations, we have determined that (1.6) is not equivalent to any of Boyd's identities, and it also does not appear to reduce to a Mahler measure formula.

Based on the computations in this paper, it is probably safe to conjecture that many values of $F(b, c)$ reduce to generalized hypergeometric functions and Meijer $G$-functions. For instance, by (4.38), we have

$$
\frac{144}{25 \pi^{2}} F(1,4)=m\left(\frac{4}{\theta}\right)+\frac{1}{4 \pi^{2}} \operatorname{Im}\left(G_{3,3}^{3,2}\left(\left.\theta^{2}\right|_{0,0,0} ^{\frac{1}{2}, \frac{1}{2}, 1}\right)\right)
$$

where $m(k)$ is defined in (3.1), and $\theta \approx 1.93+1.79 i$ is algebraic. We will briefly describe why $G$-functions appear in this context. It is often possible to show that lattice sums satisfy Picard-Fuchs equations with respect to modular parameters. If the standard solution to the equation (typically a ${ }_{4} F_{3}$ function), is analytic over the same domain as the lattice sum, then they can be equated (for an example see (4.15) ). On the other hand, if the two functions have different domains of analyticity, then the lattice sum will equate to a piecewise-defined function, which incorporates a second solution of the differential equation (sometimes a Meijer G-function). One of our main results is such a formula for $F(1,1,1, x)$, which holds whenever $x>0$ (see (4.26) ). We will also present explicit formulas for $F(1,4), F(2,2), F(1,1,2,4)$, and $F(1,2,4,4)$. Additionally, we will recover formulas for $F(1,1), F(1,2)$, and $F(1,3)$, which were first proved in $[22$.

Therefore, we will briefly outline the approach contained in Sections 4, 5, and 6, Identities such as those presented in Sections 2 and 3 require the reduction of fourdimensional sums to one-dimensional sums. If possible, the first step is a reduction to a two-dimensional sum. For instance, the left-hand side of (1.6) becomes

$$
F(1,5,5,5)=16^{2} \sum_{\substack{n=-\infty \\ k=0}}^{\infty} \frac{(-1)^{n+k}(2 k+1)}{\left((6 n+1)^{2}+15(2 k+1)^{2}\right)^{2}} .
$$


These sorts of transformations follow from well-known $q$-series results, and are quite rare. Equation (1.7) is a consequence of the following corollary to the Jacobi triple product:

$$
q^{2} \prod_{n=1}^{\infty}\left(1-q^{3 n}\right)\left(1-q^{15 n}\right)^{3}=\sum_{\substack{n=-\infty \\ k=0}}^{\infty}(-1)^{n+k}(2 k+1) q^{\frac{15(2 k+1)^{2}+(6 n+1)^{2}}{8}}
$$

Notice that equation (1.8) gives an example of a lacunary modular form [13]. The two-dimensional lattice sums are then evaluated using Ramanujan's theories of elliptic functions and modular equations. Several of these calculations are quite involved.

While the main goal of this research was to find formulas for lattice sums, we have also proved several new relations between modular forms and Mahler measures. Section 7 summarizes identities between Mahler measures and Mellin transforms of non-multiplicative modular forms. Additionally, in Section 8, we have reformulated a conjecture concerning a higher Mahler measure.

\section{Summary of Boyd's conjectures for $F(b, c)$}

In this section we will summarize a variety of explicit formulas relating four-dimensional lattice sums to Mahler measures of polynomials. Most of these results are only conjectures, although numerical calculations can be used to verify them to any degree of accuracy. Our first step will be to invoke the modularity theorem to find explicit formulas for $L$-functions of elliptic curves with conductors $N \in\{11,14,15,20,24,27,32,36\}$. The following theorem is an easy consequence of a paper due to Martin and Ono [20]:

Theorem 2.1. Suppose that $E_{N}$ is an elliptic curve of conductor $N$, then

$$
L\left(E_{N}, 2\right)=F(b, c)
$$

for the following values of $N$ and $(b, c)$ :

\begin{tabular}{|c|c|}
\hline$N$ & $(b, c)$ \\
\hline 11 & $(1,11)$ \\
14 & $(2,7)$ \\
15 & $(3,5)$ \\
20 & $(1,5)$ \\
24 & $(2,3)$ \\
27 & $(1,3)$ \\
32 & $(1,2)$ \\
36 & $(1,1)$ \\
\hline
\end{tabular}

Proof. We are interested in cases where cusp forms of elliptic curves equal the product of four eta functions. Such equalities are consequences of the modularity 
theorem. An exhaustive list of all such cusp forms is provided in [20]. By inspection of that list, the eta product associated with $E_{N}$ will have the form

$$
g(q):=q \prod_{n=1}^{\infty}\left(1-q^{A n}\right)\left(1-q^{A b n}\right)\left(1-q^{A c n}\right)\left(1-q^{A b c n}\right)
$$

where $(1+b)(1+c) A=24$. Recalling Euler's pentagonal number theorem

$$
\prod_{n=1}^{\infty}\left(1-q^{n}\right)=\sum_{n=-\infty}^{\infty}(-1)^{n} q^{n(3 n+1) / 2}
$$

this becomes

$$
g(q)=\sum_{n_{i}=-\infty}^{\infty}(-1)^{n_{1}+n_{2}+n_{3}+n_{4}} q^{\frac{A\left(6 n_{1}+1\right)^{2}+A b\left(6 n_{2}+1\right)^{2}+A c\left(6 n_{3}+1\right)^{2}+A b c\left(6 n_{4}+1\right)^{2}}{24}},
$$

and it follows immediately that

$$
L\left(E_{N}, 2\right)=\frac{24^{2}}{A^{2}} \sum_{n_{i}=-\infty}^{\infty} \frac{(-1)^{n_{1}+n_{2}+n_{3}+n_{4}}}{\left(\left(6 n_{1}+1\right)^{2}+b\left(6 n_{2}+1\right)^{2}+c\left(6 n_{3}+1\right)^{2}+b c\left(6 n_{4}+1\right)^{2}\right)^{2}} .
$$

Since $(1+b)(1+c)=24 / A$, the theorem follows.

Since we now have expressed several different $L$-values in terms of $F(b, c)$, it seems logical to list all of the known Mahler measures which reduce to values of that function.

Definition 2.2. The Mahler measure of an n-dimensional Laurent polynomial, $P\left(z_{1}, \ldots, z_{n}\right)$, is defined by

$$
\mathrm{m}(P):=\int_{0}^{1} \ldots \int_{0}^{1} \log \left|P\left(e^{2 \pi i t_{1}}, \ldots, e^{2 \pi i t_{n}}\right)\right| \mathrm{d} t_{1} \ldots \mathrm{d} t_{n} .
$$

Furthermore, we will use the following notation:

$$
\begin{aligned}
m(k) & :=\mathrm{m}\left(k+y+y^{-1}+z+z^{-1}\right), \\
n(k) & :=\mathrm{m}\left(y^{3}+z^{3}+1-k y z\right), \\
g(k) & :=\mathrm{m}((1+y)(1+z)(y+z)-k y z), \\
r(k) & :=\mathrm{m}((1+y)(1+z)(1+y+z)-k y z) .
\end{aligned}
$$

For convenience we have slightly altered the definitions of $n(k), g(k)$ and $r(k)$ that appeared in [17]. All of the following examples were either extracted from Boyd's paper [7], or were deduced by combining Boyd's conjectures with functional equations in [17]. While Boyd's minimal Weierstrass models often do not coincide 
with the minimal Weierstrass models in [20], the elliptic curves are isogenous, and the following results are all numerically true:

$$
\begin{aligned}
& n(3 \sqrt[3]{2})=\frac{27}{2 \pi^{2}} F(1,1) \\
& g(2)=\frac{9}{2 \pi^{2}} F(1,1) \\
& g(-4)=\frac{18}{\pi^{2}} F(1,1) \\
& m(4 i)=\frac{16}{\pi^{2}} F(1,2) \\
& m(2 \sqrt{2})=\frac{8}{\pi^{2}} F(1,2) \\
& n(-6)=\frac{81}{4 \pi^{2}} F(1,3) \\
& n(\sqrt[3]{2}) \stackrel{?}{=} \frac{25}{6 \pi^{2}} F(1,5) \\
& n(\sqrt[3]{32}) \stackrel{?}{=} \frac{40}{3 \pi^{2}} F(1,5) \\
& g(-2) \stackrel{?}{=} \frac{15}{\pi^{2}} F(1,5) \\
& g(4) \stackrel{?}{=} \frac{10}{\pi^{2}} F(1,5) \\
& r(-1)=\frac{77}{4 \pi^{2}} F(1,11) \\
& m(2) \stackrel{?}{=} \frac{6}{\pi^{2}} F(2,3) \\
& m(8) \stackrel{?}{=} \frac{24}{\pi^{2}} F(2,3) \\
& m(3 \sqrt{2}) \stackrel{?}{=} \frac{15}{\pi^{2}} F(2,3) \\
& m(i \sqrt{2}) \stackrel{?}{=} \frac{9}{\pi^{2}} F(2,3) \\
& n(-1) \stackrel{?}{=} \frac{7}{\pi^{2}} F(2,7) \\
& n(5) \stackrel{?}{=} \frac{49}{2 \pi^{2}} F(2,7) \\
& g(1) \stackrel{?}{=} \frac{7}{2 \pi^{2}} F(2,7) \\
& g(7) \stackrel{?}{=} \frac{21}{\pi^{2}} F(2,7) \\
& g(-8) \stackrel{?}{=} \frac{35}{\pi^{2}} F(2,7) \\
& m(1) \stackrel{?}{=} \frac{15}{4 \pi^{2}} F(3,5) \\
& m(3 i) \stackrel{?}{=} \frac{75}{4 \pi^{2}} F(3,5)
\end{aligned}
$$




$$
\begin{aligned}
m(5) & \stackrel{?}{=} \frac{45}{2 \pi^{2}} F(3,5) \\
m(16) & \stackrel{?}{=} \frac{165}{4 \pi^{2}} F(3,5)
\end{aligned}
$$

All of the results involving $F(1,1), F(1,2)$, and $F(1,3)$ can be deduced from Rodriguez-Villegas's paper [22. In particular, those Mahler measures can be written in terms of two-dimensional Eisenstein-Kronecker series, and then the results follow from Deuring's theorem.

\section{Summary of rational hypergeometric formu- las for $F(b, c)$}

In this section we will translate almost all of the known Mahler measures for $F(b, c)$ into hypergeometric functions. In Corollary 4.7 we will also prove that similar expressions exist for both $F(2,2)$ and $F(1,4)$, even though those sums are apparently unrelated to the theory of elliptic curves.

Theorem 3.1. We can express $m(k), n(k)$, and $g(k)$ in terms of generalized hypergeometric functions for most values of $k$ :

$$
\begin{aligned}
& m(k)=\operatorname{Re}\left(\log (k)-\frac{2}{k^{2}}{ }_{4} F_{3}\left(\frac{3}{2}, \frac{3}{2}, 1,1, \frac{16}{2,2,2} ; \frac{k^{2}}{2}\right)\right),
\end{aligned}
$$

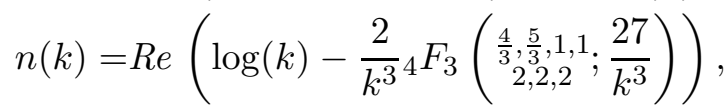

$$
\begin{aligned}
& 3 g(k)=\operatorname{Re}\left(\log \left(\frac{(4+k)(k-2)^{4}}{k^{2}}\right)-\frac{2 k^{2}}{(4+k)^{3}}{ }_{4} F_{3}\left(\begin{array}{c}
\frac{4}{3}, \frac{5}{3}, 1,1 \\
2,2,2
\end{array} ; \frac{27 k^{2}}{(4+k)^{3}}\right)\right. \\
& \left.-\frac{8 k}{(k-2)^{3}}{ }_{4} F_{3}\left(\begin{array}{c}
\frac{4}{3}, \frac{5}{3}, 1,1 \\
2,2,2
\end{array} ; \frac{27 k}{(k-2)^{3}}\right)\right) \text {. }
\end{aligned}
$$

Equation (3.1) is valid in $\mathbb{C} \backslash\{0\}$, while (3.2) and (3.3) are true for $|k|$ sufficiently large, (3.3) also holds in $\mathbb{R} \backslash[-4,2]$.

In certain cases we can reduce these hypergeometric functions further. Suppose that $k \in \mathbb{R} \backslash\{0\}$, then

$$
\operatorname{Re}\left(\log (k)-\frac{2}{k^{2}}{ }_{4} F_{3}\left(\begin{array}{c}
\frac{3}{2}, \frac{3}{2}, 1,1 \\
2,2,2
\end{array} ; \frac{16}{k^{2}}\right)\right)=\operatorname{Re}\left(\frac{|k|}{4}{ }_{3} F_{2}\left(\begin{array}{c}
\frac{1}{2}, \frac{1}{2}, \frac{1}{2} \\
1, \frac{3}{2}
\end{array} ; \frac{k^{2}}{16}\right)\right),
$$

and

$$
\begin{aligned}
& \operatorname{Re}\left(\log (k)-\frac{2}{k^{3}}{ }_{4} F_{3}\left(\begin{array}{c}
\frac{4}{3}, \frac{5}{3}, 1,1 \\
2,2,2
\end{array} ; \frac{27}{k^{3}}\right)\right)=s(k) \operatorname{Re}\left(A k_{3} F_{2}\left(\begin{array}{c}
\frac{1}{3}, \frac{1}{3}, \frac{1}{3} \\
\frac{2}{3}, \frac{4}{3}
\end{array} ; \frac{k^{3}}{27}\right)\right. \\
& \left.+B k_{3}^{2} F_{2}\left(\begin{array}{c}
\frac{2}{3}, \frac{2}{3}, \frac{2}{3} \\
\frac{4}{3}, \frac{5}{3} ; \frac{k^{3}}{27}
\end{array}\right)\right)
\end{aligned}
$$

where $A=\frac{\sqrt[3]{2} \Gamma\left(\frac{1}{6}\right) \Gamma\left(\frac{1}{3}\right) \Gamma\left(\frac{1}{2}\right)}{8 \sqrt{3} \pi^{2}}, B=\frac{\Gamma^{3}\left(\frac{2}{3}\right)}{16 \pi^{2}}$, and $s(k)=\frac{1+3 \operatorname{sgn}(k)}{4}$. 
Proof. Equations (3.1) and (3.2) are due to Rodriguez-Villegas [22], while (3.3) was proved in [17. Kurokawa and Ochiai have examined a version of (3.4) in [16], although it can also be proved by integrating the following identity:

$$
\operatorname{Re}\left({ }_{2} F_{1}\left(\frac{1}{2}, \frac{1}{2} ; u\right)\right)=\operatorname{Re}\left(\frac{1}{\sqrt{u}}{ }_{1}^{2} F_{1}\left(\frac{1}{2}, \frac{1}{2} ; \frac{1}{u}\right)\right),
$$

which holds for $u \in(0, \infty)$. A similar argument can be used to establish (3.5). For example, when $u \in(0, \infty)$ we can integrate the following transformation:

$$
\operatorname{Re}\left({ }_{2} F_{1}\left(\frac{1}{3}, \frac{2}{3} ; \frac{1}{u}\right)\right)=\operatorname{Re}\left(\frac{9 \Gamma^{3}(2 / 3)}{4 \pi^{2}} u^{2 / 3}{ }_{2} F_{1}\left(\frac{\frac{2}{3}, \frac{2}{3}}{\frac{4}{3}} ; u\right)+\frac{1}{2} u^{1 / 3}{ }_{2} F_{1}\left(\frac{1}{3}, \frac{1}{3} ; 1-u\right)\right),
$$

with respect to $u$.

Equations (3.4) and (3.5) will often allow us to obtain convergent series expansions from divergent hypergeometric formulas. For example, applying the results of the last theorem to conjecture (2.27), we obtain formula (1.3). It is hardly coincidental that (1.3) bears a striking resemblance to a famous formula that Ramanujan discovered for Catalan's constant [1]:

$$
L\left(\chi_{-4}, 2\right)=\pi \sum_{n=0}^{\infty}\left(\begin{array}{c}
2 n \\
n
\end{array}\right)^{2} \frac{(1 / 4)^{2 n+1}}{2 n+1} .
$$

Ramanujan's formula follows easily from Boyd's evaluation of the degenerate Mahler measure $m(4)$.

The following list summarizes the identities that can be obtained by reducing the Mahler measures in the previous section to hypergeometric functions. Whenever possible, we have used equations (3.4) and (3.5) to obtain hypergeometric functions with convergent arguments. Since no such expression is known for $r(-1)$, we have simply retained that Mahler measure in our list. Finally, because several Mahler measures such as $g(2)$ and $n(3 \sqrt[3]{2})$ are equivalent, this list contains fewer entries than we might expect. Once again, define

$$
A:=\frac{\sqrt[3]{2} \Gamma\left(\frac{1}{6}\right) \Gamma\left(\frac{1}{3}\right) \Gamma\left(\frac{1}{2}\right)}{8 \sqrt{3} \pi^{2}}, \quad B:=\frac{\Gamma^{3}\left(\frac{2}{3}\right)}{16 \pi^{2}},
$$

then the following results are numerically true:

$$
\begin{aligned}
& \frac{9}{2 \pi^{2}} F(1,1)=\frac{1}{9} \log (54)-\frac{1}{81}{ }_{4} F_{3}\left(\begin{array}{c}
\frac{4}{3}, \frac{5}{3}, 1,1,1 \\
2,2,2
\end{array} ; \frac{1}{2}\right), \\
& \frac{16}{\pi^{2}} F(1,2)=2 \log (2)+\frac{1}{8}{ }_{4} F_{3}\left(\begin{array}{c}
\frac{3}{2}, \frac{3}{2}, 1,1 \\
2,2,2
\end{array} ;-\frac{1}{4}\right), \\
& \frac{8}{\pi^{2}} F(1,2)=\frac{1}{\sqrt{2}} 3 F_{2}\left(\begin{array}{c}
\frac{1}{2}, \frac{1}{2}, \frac{1}{2} \\
1, \frac{3}{2}
\end{array} ; \frac{1}{2}\right), \\
& \frac{81}{4 \pi^{2}} F(1,3)=\log (6)+\frac{1}{108}{ }_{4} F_{3}\left(\begin{array}{c}
\frac{4}{3}, \frac{5}{3}, 1,1, \\
2,2,2
\end{array} ;-\frac{1}{8}\right) \text {, }
\end{aligned}
$$




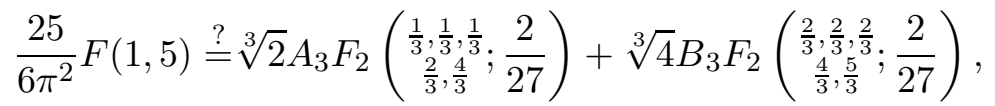

$$
\begin{aligned}
& \frac{40}{3 \pi^{2}} F(1,5) \stackrel{?}{=} \frac{5}{3} \log (2)-\frac{1}{16}{ }_{4} F_{3}\left(\begin{array}{c}
\frac{4}{3}, \frac{5}{3}, 1,1, \frac{27}{2,2,2} ; \\
32
\end{array}\right) \text {, } \\
& \frac{77}{4 \pi^{2}} F(1,11)=r(-1) \text {, } \\
& \frac{6}{\pi^{2}} F(2,3) \stackrel{?}{=} \frac{1}{2}{ }_{3} F_{2}\left(\begin{array}{c}
\frac{1}{2}, \frac{1}{2}, \frac{1}{2} \\
1, \frac{3}{2}
\end{array} ; \frac{1}{4}\right), \\
& \frac{24}{\pi^{2}} F(2,3) \stackrel{?}{=} 3 \log (2)-\frac{1}{32}{ }_{4} F_{3}\left(\underset{\frac{3}{2}}{2}, \frac{3}{2}, 1,2,2 ; \frac{1}{4}\right) \text {, } \\
& \frac{15}{\pi^{2}} F(2,3) \stackrel{?}{=} \frac{1}{2} \log (18)-\frac{1}{9} 4 F_{3}\left(\underset{\frac{3}{2}, 2,2,1,1}{2,2,2} ; \frac{8}{9}\right), \\
& \frac{9}{\pi^{2}} F(2,3) \stackrel{?}{=} \frac{1}{2} \log (2)+{ }_{4} F_{3}\left(\begin{array}{c}
\frac{3}{2}, \frac{3}{2}, 1,1 \\
2,2,2
\end{array},-8\right), \\
& \frac{7}{\pi^{2}} F(2,7) \stackrel{?}{=} \frac{A}{2}{ }_{3} F_{2}\left(\begin{array}{c}
\frac{1}{3}, \frac{1}{3}, \frac{1}{3} \\
\frac{2}{3}, \frac{4}{3}
\end{array} ;-\frac{1}{27}\right)-\frac{B}{2}{ }_{3} F_{2}\left(\begin{array}{c}
\frac{2}{3}, \frac{2}{3}, \frac{2}{3} \\
\frac{4}{3}, \frac{5}{3}
\end{array} ;-\frac{1}{27}\right), \\
& \frac{49}{2 \pi^{2}} F(2,7) \stackrel{?}{=} \log (5)-\frac{2}{125}{ }^{4} F_{3}\left(\begin{array}{c}
\frac{4}{3}, \frac{5}{3}, 1,1 \\
2,2,2
\end{array} ; \frac{27}{125}\right) \text {, } \\
& \frac{21}{\pi^{2}} F(2,7) \stackrel{?}{=} g(7) \\
& \frac{15}{4 \pi^{2}} F(3,5) \stackrel{?}{=} \frac{1}{4}{ }_{3} F_{2}\left(\begin{array}{c}
\frac{1}{2}, \frac{1}{2}, \frac{1}{2} \\
1, \frac{3}{2}
\end{array} ; \frac{1}{16}\right), \\
& \frac{45}{2 \pi^{2}} F(3,5) \stackrel{?}{=} \log (5)-\frac{2}{25}{ }_{4} F_{3}\left(\frac{3}{2}, \frac{3}{2,2,1,1} ; \frac{16}{25}\right) \text {, } \\
& \frac{165}{4 \pi^{2}} F(3,5) \stackrel{?}{=} 4 \log (2)-\frac{1}{128} 4 F_{3}\left(\begin{array}{c}
\frac{3}{2}, \frac{3}{2}, 1,1 \\
2,2,2
\end{array} ; \frac{1}{16}\right) \text {, } \\
& \frac{75}{4 \pi^{2}} F(3,5) \stackrel{?}{=} \log (3)+\frac{2}{9}{ }_{4} F_{3}\left(\frac{3}{2}, \frac{3}{2}, 1,1,1 ;-\frac{16}{9}\right) \text {. }
\end{aligned}
$$

While most of these formulas remain unproven, a variety of partial results exist. For instance, identities (3.13) through (3.16) are equivalent to one another [17, formulas (3.20) through (3.23) are equivalent to one another [18, and formulas (3.6) through (3.9) follow from [22]. Additionally, Mellit and Brunault have given $K$-theoretic proofs of the formulas for $F(2,7)$ and $F(1,11)$ (see [21] and [8]).

\section{Proofs of formulas for $F(1,1), F(1,2), F(1,4)$, $F(2,2)$, and $F(1,1,1, x)$}

In the previous section we translated many of Boyd's conjectures into explicit identities between hypergeometric functions and lattice sums. This approach has two essential consequences. Not only does it eliminate any obvious connection 
with elliptic curves, but it also allows for the construction of proofs based upon series manipulation. In this section we will discuss the cases that occur when $(b, c) \in\{(1,1),(1,2),(1,4),(2,2)\}$. We will rely heavily on the $q$-series theorems contained in Ramanujan's notebooks (see [4] and [5]).

Definition 4.1. Let us recall Ramanujan's q-series notation:

$$
\begin{array}{rlrl}
\varphi(q) & :=\sum_{n=-\infty}^{\infty} q^{n^{2}}, & \psi(q) & :=\sum_{n=0}^{\infty} q^{\frac{n(n+1)}{2}}, \\
f(-q) & :=\prod_{n=1}^{\infty}\left(1-q^{n}\right), & (x ; q)_{\infty}:=\prod_{n=0}^{\infty}\left(1-x q^{n}\right) .
\end{array}
$$

When convenient, we will also employ the following notation:

$$
e_{j}:=q^{j / 24} \prod_{n=1}^{\infty}\left(1-q^{j n}\right) .
$$

Proposition 4.3 reduces the aforementioned cases of $F(b, c)$ to two-dimensional sums. Such identities exist because various eta-quotients can be written in terms theta functions. Euler's pentagonal number formula is probably the simplest such identity:

$$
e_{j}=\sum_{n=-\infty}^{\infty}(-1)^{n} q^{\frac{j(6 n+1)^{2}}{24}} .
$$

Unfortunately, similar formulas are not known (and probably do not exist) for $e_{1}^{2}$, $e_{1} e_{2}$, or $e_{1} e_{3}$ [12. This fact represents the main obstruction to proving Boyd's conjectures for $F(1,5), F(2,3), F(2,7)$, and $F(3,5)$.

Definition 4.2. We will use the following notation:

$$
\begin{aligned}
& F_{(1,2)}(x):=\sum_{\substack{n=-\infty \\
k=0}}^{\infty} \frac{(-1)^{n+k}(2 k+1)}{\left((2 k+1)^{2}+x^{2}(2 n)^{2}\right)^{2}}, \\
& F_{(1,4)}(x):=25 \sum_{n, k=-\infty}^{\infty} \frac{(-1)^{n}(3 k+1)}{\left(4(3 k+1)^{2}+x^{2}(6 n+1)^{2}\right)^{2}}, \\
& F_{(2,2)}(x):=9 \sum_{n, k=0}^{\infty} \frac{(-1)^{\frac{n(n+1)}{2}+k}(2 k+1)}{\left(2(2 k+1)^{2}+x^{2}(2 n+1)^{2}\right)^{2}} .
\end{aligned}
$$

In the next proposition we will show that each of these functions equals a value of $F(b, c)$ when $x \rightarrow 1$. Furthermore, we will also demonstrate that a two-dimensional series exists for $F(1,1,1, x)$.

Proposition 4.3. Suppose that $(b, c) \in\{(1,2),(1,4),(2,2)\}$, then

$$
F_{(b, c)}(1)=F(b, c) \text {. }
$$


Furthermore, the following series expansion is true for any $x>0$ :

$$
\frac{F(1,1,1, x)}{(3+x)^{2}}=\sum_{\substack{n=-\infty \\ k=0}}^{\infty} \frac{(-1)^{n+k}(2 k+1)}{\left(3(2 k+1)^{2}+x(6 n+1)^{2}\right)^{2}} .
$$

Proof. First notice that $F(a, b, c, d)$ has the following integral representation for all positive values of $a, b, c$, and $d$ :

$$
\frac{24^{2} F(a, b, c, d)}{(a+b+c+d)^{2}}=\int_{0}^{1} \int_{0}^{q_{1}} e_{a} e_{b} e_{c} e_{d} \frac{\mathrm{d} q}{q} \frac{\mathrm{d} q_{1}}{q_{1}} .
$$

Taking note of the following identities:

$$
\begin{aligned}
e_{x} e_{1}^{3} & =\left(e_{x}\right) e_{1}^{3}, \\
e_{1}^{2} e_{2}^{2} & =\left(\frac{e_{1}^{2}}{e_{2}}\right) e_{2}^{3}, \\
e_{1}^{2} e_{4}^{2} & =\left(\frac{e_{1}^{2} e_{4}^{2}}{e_{2}}\right) e_{2}, \\
e_{1} e_{2}^{2} e_{4} & =\left(\frac{e_{1} e_{4}}{e_{2}}\right) e_{2}^{3},
\end{aligned}
$$

and then employing well known series expansions:

$$
\begin{aligned}
\frac{e_{1}^{2}}{e_{2}} & =\sum_{n=-\infty}^{\infty}(-1)^{n} q^{n^{2}}, \\
\frac{e_{1} e_{4}}{e_{2}} & =\sum_{n=0}^{\infty}(-1)^{\frac{n(n+1)}{2}} q^{\frac{(2 n+1)^{2}}{8}}, \\
e_{j} & =\sum_{n=-\infty}^{\infty}(-1)^{n} q^{\frac{j(6 n+1)^{2}}{24}}, \\
e_{j}^{3} & =\sum_{n=0}^{\infty}(-1)^{n}(2 n+1) q^{\frac{j(2 n+1)^{2}}{8}}, \\
\frac{e_{1}^{2} e_{4}^{2}}{e_{2}} & =\sum_{n=-\infty}^{\infty}(3 n+1) q^{\frac{(3 n+1)^{2}}{3}},
\end{aligned}
$$

we recover equations (4.5) and (4.4) in every case.

We will use the next proposition to reduce each of the two-dimensional sums to a $q$-series. Then, in Theorem 4.6, we will reduce each $q$-series to an integral of hypergeometric functions. In certain special cases those integrals translate into identities involving Mahler measures. 
Proposition 4.4. Let $\chi_{-3}(k)$ and $\chi_{-4}(k)$ denote Legendre symbols modulo three and four, and assume that $x>0$.

If $q=e^{-\pi x / \sqrt{12}}$ and $\omega=e^{\pi i / 6}$, then

$$
\frac{F\left(1,1,1, x^{2}\right)}{\left(3+x^{2}\right)^{2}}=\frac{\pi^{2}}{72 x} \sum_{k=1}^{\infty} k \chi_{-4}(k) \log \left|\frac{1+\omega q^{k}}{1-\omega q^{k}}\right| \text {. }
$$

If $q=e^{-\pi x}$, then

$$
F_{(1,2)}(x)=-\frac{\pi^{2}}{32 x}\left(\log (q)+4 \sum_{k=1}^{\infty} k \chi_{-4}(k) \log \left(1+q^{k}\right)\right) .
$$

If $q=e^{-\pi x / 3}$ and $\omega=e^{\pi i / 6}$, then

$$
F_{(1,4)}(x)=\frac{25 \pi^{2}}{72 x} \sum_{k=1}^{\infty} k \chi_{-3}(k) \log \left|\frac{1+\omega q^{k}}{1-\omega q^{k}}\right| .
$$

If $q=e^{-\pi x / \sqrt{8}}$ and $\omega=e^{\pi i / 4}$, then

$$
F_{(2,2)}(x)=\frac{9 \pi^{2}}{32 x} \sum_{k=1}^{\infty} k \chi_{-4}(k) \log \left|\frac{1+\omega q^{k}}{1-\omega q^{k}}\right| .
$$

Proof. All of the proofs are very similar, so we will only prove (4.11) in detail. First notice that

$$
\begin{aligned}
& \frac{F\left(1,1,1, x^{2}\right)}{\left(3+x^{2}\right)^{2}}= \frac{1}{144} \sum_{\substack{n=-\infty \\
k=0}}^{\infty} \frac{(-1)^{n+k}(2 k+1)}{\left((k+1 / 2)^{2}+\frac{x^{2}}{12}(6 n+1)^{2}\right)^{2}}, \\
&=\frac{\pi^{2}}{144} \int_{0}^{\infty} u\left(\sum_{k=0}^{\infty}(-1)^{k}(2 k+1) e^{-\pi(k+1 / 2)^{2} u}\right) \\
& \times\left(\sum_{n=-\infty}^{\infty}(-1)^{n} e^{-\pi(6 n+1)^{2} x^{2} u / 12}\right) \mathrm{d} u .
\end{aligned}
$$

Next, by the involution for the weight $3 / 2$ theta function:

$$
\sum_{k=0}^{\infty}(-1)^{k}(2 k+1) e^{-\pi(k+1 / 2)^{2} u}=\frac{1}{u^{3 / 2}} \sum_{k=0}^{\infty}(-1)^{k}(2 k+1) e^{-\pi(k+1 / 2)^{2} \frac{1}{u}},
$$

this becomes

$$
\frac{F\left(1,1,1, x^{2}\right)}{\left(3+x^{2}\right)^{2}}=\frac{\pi^{2}}{144} \sum_{\substack{n=-\infty \\ k=0}}^{\infty}(-1)^{n+k}(2 k+1) \int_{0}^{\infty} u^{-1 / 2} e^{-\frac{\pi(k+1 / 2)^{2}}{u}-\frac{\pi(6 n+1)^{2} x^{2}}{12} u} \mathrm{~d} u .
$$


The following integral holds whenever $A, B \in \mathbb{R}$ and $|A| \neq 0$ :

$$
\int_{0}^{\infty} u^{-1 / 2} e^{-\pi\left(A^{2} u+\frac{B^{2}}{u}\right)} \mathrm{d} u=\frac{e^{-2 \pi|A B|}}{|A|}
$$

and therefore

$$
\begin{aligned}
\frac{F\left(1,1,1, x^{2}\right)}{\left(3+x^{2}\right)^{2}} & =\frac{\pi^{2}}{24 \sqrt{3} x} \sum_{\substack{n=-\infty \\
k=0}}^{\infty}(-1)^{n+k} \frac{(2 k+1)}{|6 n+1|} e^{-\pi(2 k+1)|6 n+1| x / \sqrt{12}} \\
& =\frac{\pi^{2}}{24 \sqrt{3} x} \sum_{k=1}^{\infty} k \chi_{-4}(k) \sum_{n=-\infty}^{\infty} \frac{(-1)^{n}}{|6 n+1|} q^{k|6 n+1|} \\
& =\frac{\pi^{2}}{72 x} \sum_{k=1}^{\infty} k \chi_{-4}(k) \log \left|\frac{1+\omega q^{k}}{1-\omega q^{k}}\right|
\end{aligned}
$$

where $\omega=e^{\pi i / 6}$ and $q=e^{-\pi x / \sqrt{12}}$.

At this point, a hypergeometric formula for $F_{(1,2)}(x)$ can be recovered. By combining equation (4.12) with formulas (2-9) and (2-16) in [17, it is possible to show that if $q=e^{-\pi x}$, then

$$
F_{(1,2)}(x)=\frac{\pi^{2}}{16 x} m\left(\frac{i f^{4}(-q)}{\sqrt{q} f^{4}\left(-q^{4}\right)}\right) .
$$

In the next section we will use values of class invariants to deduce explicit examples from (4.15). Unfortunately, we will require another theorem to obtain useful results on the other lattice sums.

Theorem 4.5. In this theorem we will always assume that $x>0$. If $q=e^{-\pi x / \sqrt{12}}$, then

$$
\frac{F\left(1,1,1, x^{2}\right)}{\left(3+x^{2}\right)^{2}}=\frac{\pi^{2}}{24 \sqrt{3} x} \operatorname{Im}\left(\int_{0}^{i q} \frac{f^{9}\left(-u^{3}\right)}{f^{3}(-u)} \mathrm{d} u\right) .
$$

If $q=e^{-\pi x}$, then

$$
F_{(1,2)}(x)=\frac{\pi^{3}}{32}-\frac{\pi^{2}}{16 x} \int_{0}^{q} \frac{\varphi^{2}(-u) \varphi^{4}(u)-1}{u} \mathrm{~d} u .
$$

If $q=e^{-\pi x / 3}$ and $\rho=e^{2 \pi i / 3}$, then

$$
F_{(1,4)}(x)=\frac{25 \pi^{2}}{36 x} \operatorname{Im}\left(\int_{0}^{\rho q} \varphi^{2}(u) \psi^{4}\left(u^{2}\right) \mathrm{d} u\right) .
$$

If $q=e^{-\pi x / \sqrt{8}}$, then

$$
F_{(2,2)}(x)=\frac{9 \pi^{2}}{32 \sqrt{2} x} \int_{0}^{q} \varphi\left(-u^{2}\right) \varphi\left(u^{4}\right)\left(3 \psi^{4}\left(-u^{2}\right)-\psi^{4}\left(u^{2}\right)\right) \mathrm{d} u .
$$


Proof. Equations (4.17) and (4.18) have similar proofs, so we will only prove the latter identity. Notice that (4.13) can be rearranged to obtain

$$
\begin{aligned}
\frac{72 x}{25 \pi^{2}} F_{(1,4)}(x) & =\operatorname{Re}\left(\int_{0}^{q} \sum_{k=1}^{\infty} k^{2} \chi_{-3}(k) \frac{2 \omega u^{k}}{1-\omega^{2} u^{2 k}} \frac{\mathrm{d} u}{u}\right) \\
& =\sqrt{3} \int_{0}^{q} \sum_{k=1}^{\infty} k^{2} \chi_{-3}(k)\left(\frac{u^{k}-u^{5 k}}{1+u^{6 k}}\right) \frac{\mathrm{d} u}{u} \\
& =\operatorname{Im}\left(2 \int_{0}^{\rho q} \sum_{k=1}^{\infty} k^{2}\left(\frac{u^{k}-u^{3 k}+u^{5 k}}{1+u^{6 k}}\right) \frac{\mathrm{d} u}{u}\right) \\
& =\operatorname{Im}\left(2 \int_{0}^{\rho q} \sum_{k=1}^{\infty} \frac{k^{2} u^{k}}{1+u^{2 k}} \frac{\mathrm{d} u}{u}\right),
\end{aligned}
$$

where $\rho=e^{2 \pi i / 3}$. Combining entries 10.1, 11.3, and 17.2 in Chapter 17 of [4], we deduce that for $|u|<1$ :

$$
\sum_{k=1}^{\infty} \frac{k^{2} u^{k}}{1+u^{2 k}}=u \varphi^{2}(u) \psi^{4}\left(u^{2}\right)
$$

which completes the proof of (4.18).

The proofs of equations (4.16) and (4.19) will require the following formula:

$$
\operatorname{Im}(g(i u, t))=\frac{1}{t} \sum_{k=0}^{\infty}(-1)^{k} \frac{(2 k+1)^{2} u^{2 k+1}}{1+u^{2(2 k+1)}}\left(t^{2 k+1}-t^{-(2 k+1)}\right),
$$

where

$$
g(u, t)=\frac{(u ; u)_{\infty}^{6}\left(t^{-2} u ; u\right)_{\infty}\left(t^{2} ; u\right)_{\infty}}{\left(t^{-1} u ; u\right)_{\infty}^{4}(t ; u)_{\infty}^{4}}
$$

Equation (4.20) is a direct consequence of identity (14.2.9) in [3], which follows from product expansions for the Weierstrass $\wp$-function [9].

Rearranging equation (4.11) we have

$$
\begin{aligned}
\frac{72 x}{\pi^{2}} \frac{F\left(1,1,1, x^{2}\right)}{\left(3+x^{2}\right)^{2}} & =\operatorname{Re}\left(\int_{0}^{q} \sum_{k=0}^{\infty}(-1)^{k}(2 k+1)^{2} \frac{2 \omega u^{2 k+1}}{1-\omega^{2} u^{2(2 k+1)}} \frac{\mathrm{d} u}{u}\right) \\
& =\sqrt{3} \int_{0}^{q} \sum_{k=0}^{\infty}(-1)^{k}(2 k+1)^{2} \frac{u^{2 k+1}-u^{5(2 k+1)}}{1+u^{6(2 k+1)}} \frac{\mathrm{d} u}{u}
\end{aligned}
$$

Applying (4.20) after letting $u \rightarrow u^{3}$ and $t \rightarrow u^{-2}$, transforms this last integral into

$$
\frac{24 \sqrt{3} x}{\pi^{2}} \frac{F\left(1,1,1, x^{2}\right)}{\left(3+x^{2}\right)^{2}}=\operatorname{Im}\left(\int_{0}^{i q} \frac{f^{5}\left(-u^{2}\right) f^{4}\left(-u^{3}\right) f\left(-u^{6}\right)}{f^{4}(-u)} \mathrm{d} u\right) .
$$


By the following eta function identity:

$$
\frac{f^{5}\left(-u^{2}\right) f^{4}\left(-u^{3}\right) f\left(-u^{6}\right)}{f^{4}(-u)}=\frac{f^{9}\left(-u^{3}\right)}{f^{3}(-u)}+u \frac{f^{9}\left(-u^{6}\right)}{f^{3}\left(-u^{2}\right)}
$$

this becomes

$$
\begin{aligned}
\frac{24 \sqrt{3} x}{\pi^{2}} \frac{F\left(1,1,1, x^{2}\right)}{\left(3+x^{2}\right)^{2}} & =\operatorname{Im}\left(\int_{0}^{i q} \frac{f^{9}\left(-u^{3}\right)}{f^{3}(-u)} \mathrm{d} u\right)-\operatorname{Im}\left(\int_{0}^{q} u \frac{f^{9}\left(u^{6}\right)}{f^{3}\left(u^{2}\right)} \mathrm{d} u\right) \\
& =\operatorname{Im}\left(\int_{0}^{i q} \frac{f^{9}\left(-u^{3}\right)}{f^{3}(-u)} \mathrm{d} u\right)-0,
\end{aligned}
$$

which completes the proof of (4.16). Although we will not elaborate on the proof of (4.21) here, it suffices to say that it follows from algebraic transformations for the hypergeometric function.

The proof of (4.19) follows the same lines, but requires a few extra steps. Proceeding as before, we find that

$$
\begin{aligned}
\frac{32 x}{9 \pi^{2}} F_{(2,2)}(x) & =\operatorname{Re}\left(\int_{0}^{q} \sum_{k=0}^{\infty}(-1)^{k}(2 k+1)^{2} \frac{2 \omega u^{2 k+1}}{1-\omega^{2} u^{2(2 k+1)}} \frac{\mathrm{d} u}{u}\right) \\
& =\int_{0}^{q} \sum_{k=0}^{\infty}(-1)^{k}(2 k+1)^{2} \frac{u^{2 k+1}-u^{3(2 k+1)}}{1+u^{4(2 k+1)}} \frac{\mathrm{d} u}{u} .
\end{aligned}
$$

Applying equation (4.20) after letting $u \rightarrow u^{2}$ and $t \rightarrow u^{-1}$, this becomes

$$
\frac{32 x}{9 \pi^{2}} F_{(2,2)}(x)=\operatorname{Re}\left(2 \int_{0}^{\omega q} \frac{f^{6}\left(-u^{2}\right) f\left(-u^{8}\right)}{f\left(-u^{4}\right)} \frac{1}{\left(\omega u, u^{2}\right)_{\infty}^{4}\left(\bar{\omega} u, u^{2}\right)_{\infty}^{4}} \mathrm{~d} u\right),
$$

where $\omega=e^{\pi i / 4}$. For brevity of notation let us define a new function

$$
\begin{aligned}
g(u) & :=\left(\omega u, u^{2}\right)_{\infty}\left(\bar{\omega} u, u^{2}\right)_{\infty} \\
& =\prod_{n=0}^{\infty}\left(1-\sqrt{2} u^{2 n+1}+u^{2(2 n+1)}\right) .
\end{aligned}
$$

Since (4.14) is odd with respect to $q$, our integral can be transformed into

$$
\frac{32 x}{9 \pi^{2}} F_{(2,2)}(x)=\operatorname{Re}\left(\int_{0}^{\omega q} \frac{f^{6}\left(-u^{2}\right) f\left(-u^{8}\right)}{f\left(-u^{4}\right)} \frac{g^{4}(u)+g^{4}(-u)}{g^{4}(u) g^{4}(-u)} \mathrm{d} u\right) .
$$

Next we will reduce $\left(g^{4}(u)+g^{4}(-u)\right) /(g(u) g(-u))^{4}$ to theta functions. Observe by equation (4.22) that

$$
g(u) g(-u)=\prod_{n=0}^{\infty}\left(1+u^{4(2 n+1)}\right)=\frac{\varphi\left(-u^{8}\right)}{f\left(-u^{4}\right)} .
$$


With two applications of the Jacobi triple product [4], we also have

$$
\begin{aligned}
g(u)+g(-u) & =\frac{1}{f\left(-u^{2}\right)}\left(\sum_{n=-\infty}^{\infty}(-\omega)^{n} u^{n^{2}}+\sum_{n=-\infty}^{\infty} \omega^{n} u^{n^{2}}\right) \\
& =\frac{2}{f\left(-u^{2}\right)} \sum_{n=-\infty}^{\infty}(-1)^{n} u^{16 n^{2}} \\
& =\frac{2 \varphi\left(-u^{16}\right)}{f\left(-u^{2}\right)}
\end{aligned}
$$

So finally, combining (4.24) and (4.25), we find that

$$
\frac{g^{4}(u)+g^{4}(-u)}{g^{4}(u) g^{4}(-u)}=2 \frac{f^{4}\left(-u^{4}\right)}{f^{4}\left(-u^{2}\right)}\left(8 \frac{\varphi^{4}\left(-u^{16}\right)}{\varphi^{4}\left(-u^{8}\right)}-8 \frac{\varphi^{2}\left(-u^{16}\right) \varphi\left(-u^{2}\right)}{\varphi^{3}\left(-u^{8}\right)}+\frac{\varphi^{2}\left(-u^{2}\right)}{\varphi^{2}\left(-u^{8}\right)}\right) .
$$

Recalling that $\varphi^{2}\left(-q^{16}\right)=\varphi\left(-q^{8}\right) \varphi\left(q^{8}\right)$, this becomes

$$
\begin{aligned}
\frac{g^{4}(u)+g^{4}(-u)}{g^{4}(u) g^{4}(-u)} & =2 \frac{f^{4}\left(-u^{4}\right)}{f^{4}\left(-u^{2}\right)}\left(\frac{8 \varphi^{2}\left(u^{8}\right)-8 \varphi\left(u^{8}\right) \varphi\left(-u^{2}\right)+\varphi^{2}\left(-u^{2}\right)}{\varphi^{2}\left(-u^{8}\right)}\right), \\
& =2 \frac{f^{5}\left(-u^{4}\right)}{f^{6}\left(-u^{2}\right)}\left(\frac{8 \varphi^{2}\left(u^{8}\right) \varphi\left(-u^{2}\right)-8 \varphi\left(u^{8}\right) \varphi^{2}\left(-u^{2}\right)+\varphi^{3}\left(-u^{2}\right)}{\varphi^{2}\left(-u^{8}\right)}\right),
\end{aligned}
$$

and therefore (4.23) simplifies to

$$
\begin{aligned}
\frac{32 x}{9 \pi^{2}} F_{(2,2)}(x)=\operatorname{Re}\left(2 \int_{0}^{\omega q}\right. & f^{4}\left(-u^{4}\right) f\left(-u^{8}\right) \\
& \left.\times \frac{8 \varphi^{2}\left(u^{8}\right) \varphi\left(-u^{2}\right)-8 \varphi\left(u^{8}\right) \varphi^{2}\left(-u^{2}\right)+\varphi^{3}\left(-u^{2}\right)}{\varphi^{2}\left(-u^{8}\right)} \mathrm{d} u\right) .
\end{aligned}
$$

Next, let $u \rightarrow \omega u$ to obtain

$$
\begin{aligned}
=2 \int_{0}^{q} & \frac{f^{4}\left(u^{4}\right) f\left(-u^{8}\right)}{\varphi^{2}\left(-u^{8}\right)} \\
& \times \operatorname{Re}\left(\omega\left(8 \varphi^{2}\left(u^{8}\right) \varphi\left(-i u^{2}\right)-8 \varphi\left(u^{8}\right) \varphi^{2}\left(-i u^{2}\right)+\varphi^{3}\left(-i u^{2}\right)\right)\right) \mathrm{d} u .
\end{aligned}
$$

If we recall that $\varphi\left(-i u^{2}\right)=\varphi\left(u^{8}\right)-2 i u^{2} \psi\left(u^{16}\right)$, then we are left with

$$
\begin{aligned}
\frac{32 x}{9 \pi^{2}} F_{(2,2)}(x)=\sqrt{2} \int_{0}^{q} & \frac{f^{4}\left(u^{4}\right) f\left(-u^{8}\right)}{\varphi^{2}\left(-u^{8}\right)} \\
& \times\left(\left(\varphi\left(u^{8}\right)-2 u^{2} \psi\left(u^{16}\right)\right)^{3}-4 u^{2} \varphi\left(u^{8}\right) \psi\left(u^{16}\right)\left(\varphi\left(u^{8}\right)-2 u^{2} \psi\left(u^{16}\right)\right)\right) \mathrm{d} u .
\end{aligned}
$$


In order to simplify this last formula, we will freely apply theta function identities on pages 34 and 40 of [4]. Therefore, we find that

$$
\begin{aligned}
& =\sqrt{2} \int_{0}^{q} \frac{f^{4}\left(u^{4}\right) f\left(-u^{8}\right)}{\varphi^{2}\left(-u^{8}\right)}\left(\varphi^{3}\left(-u^{2}\right)-4 u^{2} \varphi\left(u^{8}\right) \psi\left(u^{16}\right) \varphi\left(-u^{2}\right)\right) \mathrm{d} u \\
& =\sqrt{2} \int_{0}^{q} \frac{f^{4}\left(u^{4}\right) f\left(-u^{8}\right)}{\varphi^{2}\left(-u^{8}\right)} \varphi\left(-u^{2}\right)\left(\varphi^{2}\left(-u^{2}\right)-4 u^{2} \psi^{2}\left(u^{8}\right)\right) \mathrm{d} u \\
& =\frac{1}{\sqrt{2}} \int_{0}^{q} \frac{f^{4}\left(u^{4}\right) f\left(-u^{8}\right)}{\varphi^{2}\left(-u^{8}\right)} \varphi\left(-u^{2}\right)\left(3 \varphi^{2}\left(-u^{2}\right)-\varphi^{2}\left(u^{2}\right)\right) \mathrm{d} u \\
& =\frac{1}{\sqrt{2}} \int_{0}^{q} \frac{f^{4}\left(u^{4}\right) f\left(-u^{8}\right)}{\varphi^{2}\left(-u^{8}\right) \psi^{2}\left(u^{4}\right)} \varphi\left(-u^{2}\right)\left(3 \psi^{4}\left(-u^{2}\right)-\psi^{4}\left(u^{2}\right)\right) \mathrm{d} u \\
& =\frac{1}{\sqrt{2}} \int_{0}^{q} \varphi\left(u^{4}\right) \varphi\left(-u^{2}\right)\left(3 \psi^{4}\left(-u^{2}\right)-\psi^{4}\left(u^{2}\right)\right) \mathrm{d} u,
\end{aligned}
$$

which completes the proof of (4.19).

The next theorem requires the signature-three theta functions. Recall that if $\omega=e^{2 \pi i / 3}$, then the signature-three theta functions are defined by:

$$
\begin{aligned}
& a(q):=\sum_{n, m=-\infty}^{\infty} q^{m^{2}+m n+n^{2}}, \\
& b(q):=\sum_{n, m=-\infty}^{\infty} \omega^{m-n} q^{m^{2}+m n+n^{2}}, \\
& c(q):=\sum_{n, m=-\infty}^{\infty} q^{(m+1 / 3)^{2}+(m+1 / 3)(n+1 / 3)+(n+1 / 3)^{2}} .
\end{aligned}
$$

The signature-three theta functions satisfy many interesting formulas, including the following cubic relation:

$$
a^{3}(q)=b^{3}(q)+c^{3}(q)
$$

Various other properties of $a(q), b(q)$, and $c(q)$ have been catalogued in [5].

Theorem 4.6. We can reduce the two-dimensional lattice sums to integrals of hypergeometric functions.

Suppose that $q=e^{-\pi x / \sqrt{12}}$, then

$$
\frac{648 x}{\pi^{2}} \frac{F\left(1,1,1, x^{2}\right)}{\left(3+x^{2}\right)^{2}}= \begin{cases}3 \tilde{n}\left(3 \frac{a(i q)}{b(i q)}\right)+\frac{4}{\sqrt{3}} n_{2}\left(\frac{b^{3}(i q)}{a^{3}(i q)}\right) & \text { if } x \in\left(0, \frac{1}{\sqrt{5}}\right), \\ 3 \tilde{n}\left(3 \frac{a(i q)}{b(i q)}\right)+\frac{1}{\sqrt{3}} n_{2}\left(\frac{b^{3}(i q)}{a^{3}(i q)}\right) & \text { if } x \in\left(\frac{1}{\sqrt{5}}, \sqrt{5}\right), \\ \frac{1}{\sqrt{3}} n_{2}\left(\frac{b^{3}(i q)}{a^{3}(i q)}\right) & \text { if } x \in(\sqrt{5}, \infty),\end{cases}
$$

where

$$
\tilde{n}(k)=\operatorname{Re}\left(\log (k)-\frac{2}{k^{3}}{ }_{4} F_{3}\left(\begin{array}{c}
1,1, \frac{4}{3}, \frac{5}{3} \\
2,2,2
\end{array} \frac{27}{k^{3}}\right)\right),
$$


and

$$
n_{2}(k)=\operatorname{Im}\left(\int_{k}^{1} \frac{{ }_{2} F_{1}\left(\frac{1}{3}, \frac{2}{3} ; 1-u\right)}{u} \mathrm{~d} u\right) .
$$

Notice that $\tilde{n}(k)=n(k)$ whenever $|k|$ is sufficiently large.

Suppose that $q=e^{-\pi x}$ and $x>0$, then

$$
\frac{16 x}{\pi^{2}} F_{(1,2)}(x)=m\left(\frac{i f^{4}(-q)}{\sqrt{q} f^{4}\left(-q^{4}\right)}\right),
$$

where $m(k)$ is defined in (2.3).

Suppose that $q=e^{-\pi x / 3}$ and $\omega=e^{2 \pi i / 3}$, then

$$
\frac{144 x}{25 \pi^{2}} F_{(1,4)}(x)= \begin{cases}m\left(4 \frac{\varphi^{2}(\omega q)}{\varphi^{2}(-\omega q)}\right)-\frac{3}{4} m_{2}\left(\frac{\varphi^{4}(-\omega q)}{\varphi^{4}(\omega q)}\right) & \text { if } x \in\left(0, \frac{1}{\sqrt{2}}\right), \\ m\left(4 \frac{\varphi^{2}(\omega q)}{\varphi^{2}(-\omega q)}\right)+\frac{1}{4} m_{2}\left(\frac{\varphi^{4}(-\omega q)}{\varphi^{4}(\omega q)}\right) & \text { if } x \in\left(\frac{1}{\sqrt{2}}, \sqrt{2}\right), \\ \frac{1}{4} m_{2}\left(\frac{\varphi^{4}(-\omega q)}{\varphi^{4}(\omega q)}\right) & \text { if } x \in(\sqrt{2}, \infty)\end{cases}
$$

where $m(k)$ is defined in (2.3), and

$$
m_{2}(k):=\operatorname{Im}\left(\int_{k}^{1} \frac{{ }_{2} F_{1}\left(\frac{1}{2}, \frac{1}{2} ; 1-u\right)}{u} \mathrm{~d} u\right) .
$$

$$
\begin{aligned}
& \text { If } q=e^{-\pi x / \sqrt{8}} \text {, then } \\
& \qquad F_{(2,2)}(x)=\frac{9 \pi^{2}}{256 x} \int_{\frac{\varphi^{2}\left(-q^{2}\right)}{\varphi^{2}\left(q^{2}\right)}}^{1} \frac{(3 u-1)}{\sqrt{u(1-u)}} 2 F_{1}\left(\frac{1}{2}, \frac{1}{2} ; 1-u^{2}\right) \mathrm{d} u .
\end{aligned}
$$

Proof. The proof of this theorem follows from our ability to invert theta functions. First recall the classical inversion formula for the theta function:

$$
\varphi^{2}(q)={ }_{2} F_{1}\left(\frac{1}{2}, \frac{1}{2} ; 1-\frac{\varphi^{4}(-q)}{\varphi^{4}(q)}\right),
$$

which holds whenever $q \in(-1,1)$ [4]. If we use the notation $\alpha=1-\varphi^{4}(-q) / \varphi^{4}(q)$ and $z=\varphi^{2}(q)$, then many different theta functions can be expressed in terms of these two parameters. The following identities are true whenever $|q|<1$ :

$$
\begin{aligned}
\varphi(q) & =\sqrt{z} \\
\varphi(-q) & =(1-\alpha)^{1 / 4} \sqrt{z}, \\
\varphi\left(q^{2}\right) & =(1+\sqrt{1-\alpha})^{1 / 2} \sqrt{\frac{z}{2}} \\
\psi(-q) & =q^{-1 / 8}\{\alpha(1-\alpha)\}^{1 / 8} \sqrt{\frac{z}{2}}
\end{aligned}
$$




$$
\psi(q)=q^{-1 / 8} \alpha^{1 / 8} \sqrt{\frac{z}{2}}
$$

and it is also well known that

$$
\frac{\mathrm{d} \alpha}{\mathrm{d} q}=\frac{\alpha(1-\alpha) z^{2}}{q}
$$

Both (4.27) and (4.29) follow from applying these parameterizations to equations (4.17) and (4.19) respectively.

Since equation (4.30) does not hold in the entire open unit disk, we will need to generalize that result. First notice that $z$ satisfies the hypergeometric differential equation with respect to $\alpha$ :

$$
\alpha(1-\alpha) \frac{\mathrm{d}^{2} z}{\mathrm{~d} \alpha^{2}}+(1-2 \alpha) \frac{\mathrm{d} z}{\mathrm{~d} \alpha}-\frac{z}{4}=0 .
$$

We can use the relation $\frac{\mathrm{d}}{\mathrm{d} \alpha}=\frac{1}{\frac{\mathrm{d} \alpha}{\mathrm{d} q}} \times \frac{\mathrm{d}}{\mathrm{d} q}$, to show that (4.31) holds (excluding possible poles) for $|q|<1$. The most general solution of this differential equation has the form

$$
z=C_{2} F_{1}\left(\frac{1}{2}, \frac{1}{2} ; \alpha\right)+D_{2} F_{1}\left(\frac{1}{2}, \frac{1}{2} ; 1-\alpha\right),
$$

where $C$ and $D$ are undetermined constants. When $q$ lies in a neighborhood of zero, (4.30) shows that $(C, D)=(1,0)$. We can analytically continue that solution to a larger connected $q$-domain, provided that $\alpha$ (and $1-\alpha$ if $D \neq 0$ ) does not intersect the line $[1, \infty)$. In particular, the function ${ }_{2} F_{1}\left(\frac{1}{2}, \frac{1}{2} ; \alpha\right)$ has a branch cut running along $[1, \infty)$.

If we consider values of $q \in(0, \omega)$ with $\omega=e^{2 \pi i / 3}$, then $\alpha$ crosses $[1, \infty)$ at the point $q=\omega e^{-\pi \sqrt{2} / 3}$. Similarly, $1-\alpha$ intersects the branch cut at $q=\omega e^{-\pi / 3 \sqrt{2}}$. It follows that we will have to solve the hypergeometric differential equation separately on each of the three line segments. If $u=\omega e^{-\pi x / 3}$, then

$$
\varphi^{2}(u)= \begin{cases}-3 F_{1}\left(\frac{1}{2}, \frac{1}{2} ; 1-\frac{\varphi^{4}(-u)}{\varphi^{4}(u)}\right)+2 i_{2} F_{1}\left(\frac{1}{2}, \frac{1}{2} ; \frac{\varphi^{4}(-u)}{\varphi^{4}(u)}\right) & \text { if } x \in\left(0, \frac{1}{\sqrt{2}}\right), \\ { }_{2} F_{1}\left(\frac{1}{2}, \frac{1}{2} ; 1-\frac{\varphi^{4}(-u)}{\varphi^{4}(u)}\right)+2 i_{2} F_{1}\left(\frac{1}{2}, \frac{1}{2} ; \frac{\varphi^{4}(-u)}{\varphi^{4}(u)}\right) & \text { if } x \in\left(\frac{1}{\sqrt{2}}, \sqrt{2}\right), \\ { }_{2} F_{1}\left(\frac{1}{2}, \frac{1}{2} ; 1-\frac{\varphi^{4}(-u)}{\varphi^{4}(u)}\right) & \text { if } x \in(\sqrt{2}, \infty) .\end{cases}
$$

The coefficients in (4.32) can be verified from the fact that $\varphi^{2}(u)$ is analytic when $x \in(0, \infty)$. For example, we can check the continuity of the right-hand side of (4.32) by letting $u \rightarrow \omega e^{-\pi \sqrt{2} / 3}$. In that case $\alpha=1-\frac{\varphi^{4}(-u)}{\varphi^{4}(u)} \approx 5.828 \ldots$, and we have:

$$
\begin{aligned}
0 & =\varphi^{2}\left(\omega e^{-\pi \frac{\sqrt{2}+0}{3}}\right)-\varphi^{2}\left(\omega e^{-\pi \frac{\sqrt{2}-0}{3}}\right) \\
& ={ }_{2} F_{1}\left(\frac{1}{2}, \frac{1}{2} ; \alpha+i 0\right)-{ }_{2} F_{1}\left(\frac{1}{2}, \frac{1}{2} ; \alpha-i 0\right)-2 i_{2} F_{1}\left(\frac{1}{2}, \frac{1}{2} ; 1-\alpha\right) .
\end{aligned}
$$


This vanishing of this last expression follows from basic properties of the hypergeometric function (see problem 1 on page 276 of [19]), and therefore the right-hand side of (4.32) is indeed continuous at $x=\sqrt{2}$. In practice, we simply discovered (4.32) numerically.

We will use the theory of signature-three theta functions to prove equation (4.26). Recall that $c(q)$ can be expressed as an infinite product:

$$
\frac{c^{3}(q)}{27 q}=\frac{f^{9}\left(-q^{3}\right)}{f^{3}(-q)}
$$

and that the signature-three theta functions obey a differentiation formula:

$$
\frac{c^{3}(q)}{q}=\frac{a(q)}{1-\frac{c^{3}(q)}{a^{3}(q)}} \frac{\mathrm{d}}{\mathrm{d} q}\left(\frac{c^{3}(q)}{a^{3}(q)}\right) .
$$

It follows immediately that equation (4.16) reduces to

$$
\frac{F\left(1,1,1, x^{2}\right)}{\left(3+x^{2}\right)^{2}}=\frac{\pi^{2}}{648 \sqrt{3} x} \operatorname{Im}\left(\int_{0}^{i q} \frac{a(u)}{1-\frac{c^{3}(u)}{a^{3}(u)}} \frac{\mathrm{d}}{\mathrm{d} u}\left(\frac{c^{3}(u)}{a^{3}(u)}\right) \mathrm{d} u\right) .
$$

Next recall that for $|u|$ sufficiently small:

$$
a(u)={ }_{2} F_{1}\left(\frac{1}{3}, \frac{2}{3} ; \frac{c^{3}(u)}{a^{3}(u)}\right) .
$$

In order to apply (4.34) to our integral, we will need to establish a generalized inversion formula which holds for $u \in(0, i)$. The reasoning closely follows the proof of (4.32), except that $c^{3}(u) / a^{3}(u) \in[1, \infty)$ when $u=i e^{-\pi \sqrt{5 / 12}}$, and $1-$ $c^{3}(u) / a^{3}(u) \in[1, \infty)$ when $u=i e^{-\pi / \sqrt{60}}$. Suppose that $u=i e^{-\pi x / \sqrt{12}}$, then we obtain

$$
a(u)= \begin{cases}4_{2} F_{1}\left(\frac{1}{3}, \frac{2}{3} ; \frac{c^{3}(u)}{a^{3}(u)}\right)+\sqrt{3} i_{2} F_{1}\left(\frac{1}{3}, \frac{2}{3} ; 1-\frac{c^{3}(u)}{a^{3}(u)}\right) & \text { if } x \in\left(0, \frac{1}{\sqrt{5}}\right), \\ 2{ }_{2} F_{1}\left(\frac{1}{3}, \frac{2}{3} ; \frac{c^{3}(u)}{a^{3}(u)}\right)+\sqrt{3} i_{2} F_{1}\left(\frac{1}{3}, \frac{2}{3} ; 1-\frac{c^{3}(u)}{a^{3}(u)}\right) & \text { if } x \in\left(\frac{1}{\sqrt{5}}, \sqrt{5}\right), \\ { }_{2} F_{1}\left(\frac{1}{3}, \frac{2}{3} ; \frac{c^{3}(u)}{a^{3}(u)}\right) & \text { if } x \in(\sqrt{5}, \infty) .\end{cases}
$$

Finally, (4.26) follows from substituting (4.35) into (4.33) and simplifying.

Finally, we will conclude this section by summarizing the formulas that follow from setting $x=1$ in Theorem 4.6.

Corollary 4.7. The following identities are true:

$$
\begin{aligned}
\frac{27}{2 \pi^{2}} F(1,1) & =n(3 \sqrt[3]{2}), \\
\frac{16}{\pi^{2}} F(1,2) & =m(4 i),
\end{aligned}
$$




$$
\begin{aligned}
\frac{144}{25 \pi^{2}} F(1,4) & =m\left(\frac{4}{\theta}\right)+\frac{1}{4} \operatorname{Im}\left(\int_{\theta^{2}}^{1} \frac{{ }_{2} F_{1}\left(\frac{1}{2}, \frac{1}{2} ; 1-u\right)}{u} \mathrm{~d} u\right), \\
\frac{256}{9 \pi^{2}} F(2,2) & =\int_{\sqrt{2}-1}^{1} \frac{3 u-1}{\sqrt{u(1-u)}}{ }_{2} F_{1}\left(\frac{1}{2}, \frac{1}{2} ; 1-u^{2}\right) \mathrm{d} u,
\end{aligned}
$$

where $\theta=\frac{\left(4-2 t-2 t^{2}+t^{3}\right)}{4 \sqrt{2}}$, and $t=-i \sqrt[4]{12}$.

Notice that equation (4.38) involves Meijer's $G$-function disguised as a hypergeometric integral:

$$
\operatorname{Im}\left(\int_{k}^{1} \frac{{ }_{2} F_{1}\left(\frac{1}{2}, \frac{1}{2} ; 1-u\right)}{u} \mathrm{~d} u\right)=\frac{1}{\pi^{2}} \operatorname{Im}\left(G_{3,3}^{3,2}\left(\left.k\right|_{0,0,0} ^{\frac{1}{2}, \frac{1}{2}, 1}\right)\right) .
$$

This identity probably rules out the possibility of expressing $F(1,4)$ as a Mahler measure, and it also indicates that any explicit formula for $F(b, c)$ should reduce to Meijer $G$-functions in certain instances.

\section{Formulas for $F(1,1,2,4), F(1,2,4,4)$, and ad- ditional explicit examples}

In this section, we will present several additional formulas for $F(a, b, c, d)$. Since the ideas are the same as in the previous section, we will only sketch brief details of each proof.

Theorem 5.1. The following identities are true:

$$
\begin{aligned}
\frac{144 \sqrt{2}}{121 \pi^{2}} F(1,2,4,4) & =\frac{1}{3} n\left(3\left(\frac{4}{2+17 \sqrt{2}-9 \sqrt{6}}\right)^{1 / 3}\right)-\frac{1}{6} n\left(3\left(\frac{4}{2+\sqrt{2}}\right)^{1 / 3}\right) \\
\frac{27}{\sqrt{2} \pi^{2}} F(1,1,2,4) & =n\left(3\left(\frac{4}{2-17 \sqrt{2}+9 \sqrt{6}}\right)^{1 / 3}\right)-n\left(3\left(\frac{4}{2-\sqrt{2}}\right)^{1 / 3}\right) .
\end{aligned}
$$

Proof. The proof of this theorem requires the following identities:

$$
\begin{aligned}
& e_{1}^{2} e_{2} e_{4}=\left(\frac{e_{1}^{2} e_{4}^{2}}{e_{2}}\right)\left(\frac{e_{2}^{2}}{e_{4}}\right)=\sum_{n, k=-\infty}^{\infty}(-1)^{k}(3 n+1) q^{\frac{(3 n+1)^{2}+6 k^{2}}{3}}, \\
& e_{1} e_{2} e_{4}^{2}=\left(\frac{e_{1}^{2} e_{4}^{2}}{e_{2}}\right)\left(\frac{e_{2}^{2}}{e_{1}}\right)=\sum_{\substack{n=-\infty \\
k=0}}^{\infty}(3 n+1) q^{\frac{8(3 n+1)^{2}+3(2 k+1)^{2}}{24}}
\end{aligned}
$$


By an argument similar to the one in Proposition 4.4, we can prove that

$$
\begin{aligned}
F(1,1,2,4) & =\sum_{n, k=-\infty}^{\infty} \frac{(-1)^{k}(3 n+1)}{\left((3 n+1)^{2}+6 k^{2}\right)^{2}} \\
& =-\frac{\sqrt{2} \pi^{2}}{81}\left(\log \left(q^{4}\right)+9 \sum_{n=1}^{\infty} n \chi_{-3}(n) \log \left(1+q^{4 n}\right)\right),
\end{aligned}
$$

and

$$
\begin{aligned}
F(1,2,4,4) & =\frac{121}{2} \sum_{n, k=-\infty}^{\infty} \frac{(3 n+1)}{\left(8(3 n+1)^{2}+3(2 k+1)^{2}\right)^{2}} \\
& =\frac{121 \pi^{2}}{288 \sqrt{2}} \sum_{n=1}^{\infty} n \chi_{-3}(n) \log \left(\frac{1+q^{n}}{1-q^{n}}\right)
\end{aligned}
$$

where $q=e^{-\pi / \sqrt{6}}$. Both of these $q$-series can be evaluated in terms of $n(k)$ by the results of Rodriguez-Villegas (see [17] for details).

If we consider additional examples involving $F\left(1,1,1, x^{2}\right)$, then we can establish many interesting formulas by setting $x=\sqrt{a / b}$. For instance, if $x \in\left\{1, \frac{1}{\sqrt{5}}\right\}$, we have $\operatorname{Im}\left(b^{3}(i q) / a^{3}(i q)\right)=0$, and as a result it is possible to show that the $n_{2}$ term in (4.26) vanishes.

Theorem 5.2. Let $\phi=\frac{1+\sqrt{5}}{2}, y=\sqrt[3]{2} e^{\pi i / 3}$, and suppose that $z \approx-.58+.56 i$ is a root of the equation $\left(z^{2}+3 z+1\right)^{3}-2\left(z^{6}+1\right)=0$, then

$$
\begin{aligned}
F(1,1) & =\frac{2 \pi^{2}}{27} n(3 \sqrt[3]{2}), \\
F\left(1,1,1, \frac{1}{5}\right) & =\frac{32 \pi^{2}}{135 \sqrt{5}} \tilde{n}\left(\frac{3}{\sqrt[3]{\phi}}\right), \\
F(1,1,1,5) & \stackrel{?}{=} \frac{4 \pi^{2}}{27 \sqrt{5}} n(-3 \sqrt[3]{\phi}), \\
F(1,1,1,9) & =\frac{2 \pi^{2}}{27 \sqrt{3}} n_{2}\left(\frac{9}{2\left(1+y+3 y^{2}\right)}\right), \\
F(1,1,1,25) & =\frac{98 \pi^{2}}{405 \sqrt{3}} n_{2}\left(\frac{1}{1+z^{6}}\right) .
\end{aligned}
$$

Notice that the result in the introduction, (1.6), follows from combining (5.4) with (3.5). We have also stated (5.5) as a conjecture, because the result presumably follows from (4.26) when $x \rightarrow \sqrt{5}$, but we still have not found a completely rigorous proof of that fact. Finally, we will conclude this section with two additional identities. If we set $q=e^{-\frac{\pi}{2 \sqrt{3}}}$ and $\omega=e^{\pi i / 4}$, then

$$
\frac{24^{2} \sqrt{2}}{19^{2} \pi^{2}} F(1,2,8,8)=\sum_{n=1}^{\infty} n \chi_{-3}(n) \log \left|\frac{1+\omega q^{n}}{1-\omega q^{n}}\right|,
$$




$$
\frac{72 \sqrt{2}}{49 \pi^{2}} \mathrm{~F}(1,1,4,8)=\sum_{n=1}^{\infty} n \chi_{-3}(n) \log \left|\frac{1+\omega q^{2 n}}{1-\omega q^{2 n}}\right| .
$$

Unfortunately, we have not been able to reduce these last two $q$-series to known functions. These $q$-expansions follow from combining $e_{1} e_{2} e_{8}^{2}=\left(\frac{e_{2}^{2} e_{8}^{2}}{e_{4}}\right)\left(\frac{e_{1} e_{4}}{e_{2}}\right)$ and $e_{1}^{2} e_{4} e_{8}=\left(\frac{e_{1}^{2} e_{4}^{2}}{e_{2}}\right)\left(\frac{e_{2} e_{8}}{e_{4}}\right)$, with equations (4.7) and (4.10).

\section{Remarks on $F(1,3), F(2,9), F(5,9)$ and higher values of $F(a, b, c, d)$}

In this section, we will briefly demonstrate how to reduce several higher values of $F(a, b, c, d)$ to known functions. Our proof of the $F(1,3)$ formula will be instructive. Recall that Rodriguez-Villegas showed that

$$
\frac{4 \pi^{2}}{81} n(-6)=\operatorname{Re}\left(\frac{1}{2} \sum_{\substack{m, n \in \mathbb{Z} \\(m, n) \neq(0,0)}} \frac{\chi_{-3}(n)}{\left(3\left(\frac{1+i \sqrt{3}}{2}\right) m+n\right)^{2}\left(3\left(\frac{1-i \sqrt{3}}{2}\right) m+n\right)}\right),
$$

and then used Deuring's theorem to equate this Eisenstein series to the $L$ series of a CM elliptic curve of conductor 27 [22, p. 32]. A different proof could have been constructed from numerically observing that

$$
\begin{aligned}
& q \prod_{n=1}^{\infty}\left(1-q^{3 n}\right)^{2}\left(1-q^{9 n}\right)^{2} \\
& \quad=\frac{1}{4} \sum_{j=1}^{2} \chi_{-3}(j) \sum_{n, m=-\infty}^{\infty}((6 m+j)+3(6 n+j)) q^{\frac{(6 m+j)^{2}+3(6 n+j)^{2}}{4}} .
\end{aligned}
$$

The modularity theorem implies that $e_{3}^{2} e_{9}^{2}$ is associated to the correct elliptic curve, hence the Mellin transform of the left-hand side of equation (6.2) will equal $L(E, s)$. Since the Mellin transform (at $s=2$ ) of the right-hand side trivially equals the righthand side of equation (6.1), it just remains to prove (6.2). By applying limiting cases

of the triple and quintuple product identities, we can show that equation (6.2) is equivalent to an identity between eta functions:

$$
4 e_{3}^{2} e_{9}^{2}=\left(\frac{e_{6}^{5} e_{36} e_{54}^{2}}{e_{12}^{2} e_{18} e_{108}}\right)+3\left(\frac{e_{12} e_{18}^{7}}{e_{6} e_{36}^{3}}\right)-2\left(\frac{e_{3}^{2} e_{12}^{2} e_{18}^{2} e_{27} e_{108}}{e_{6} e_{9} e_{36} e_{54}}\right)-6\left(\frac{e_{6}^{2} e_{9}^{3} e_{36}^{3}}{e_{3} e_{12} e_{18}^{2}}\right) .
$$

Identities between modular forms, such as (6.3), are usually established by checking that both sides of a formula have the same McLauren series expansion for sufficiently many terms. If we use the inversion formula for the eta function, then (6.3) can also be viewed as an example of a mixed modular equation [4]. Of course, the main difficulty of extending this type of approach, is to actually find the necessary modular equations. Since the first version of this paper appeared, it has become clear that this method also applies to $F(2,9), F(5,9)$ and $F(4,7,7,28)$ [24]. 


\section{New Mahler measures, and the $L$-series of an irrational modular form}

In this section we will use values of class invariants to deduce some explicit formulas for Mahler measures. Recall that if $q=e^{-\pi \sqrt{m}}$, then the class invariants are defined by

$$
g_{m}:=2^{-1 / 4} q^{-1 / 24}\left(q ; q^{2}\right)_{\infty}, \quad G_{m}:=2^{-1 / 4} q^{-1 / 24}\left(-q ; q^{2}\right)_{\infty} .
$$

It is a classical fact that $G_{m}$ and $g_{m}$ are algebraic numbers whenever $m \in \mathbb{Q}$, and that they satisfy the following algebraic relation:

$$
\left(g_{m} G_{m}\right)^{8}\left(G_{m}^{8}-g_{m}^{8}\right)=\frac{1}{4} .
$$

Since most tables only contain values of $g_{m}$ when $m$ is even, and $G_{m}$ when $m$ is odd, our calculations will require (7.1).

Theorem 7.1. Suppose that $m \in \mathbb{N}$, then

$$
m\left(8 i g_{m}^{8} G_{m}^{4}\right)=\frac{16 \sqrt{m}}{\pi^{2}} \sum_{n=1}^{\infty} \frac{b_{n}}{n^{2}},
$$

where

$$
\sum_{n=1}^{\infty} b_{n} q^{n}=\frac{e_{8}^{3} e_{4 m}^{2}}{e_{8 m}} .
$$

The following table gives evaluations of $8 g_{m}^{8} G_{m}^{4}$, and states whether or not $b_{n}$ is multiplicative:

\begin{tabular}{|c|c|c|}
\hline$m$ & $8 g_{m}^{8} G_{m}^{4}$ & $b_{n}$ multiplicative? \\
\hline 1 & 4 & Yes \\
2 & $4 \sqrt{2+2 \sqrt{2}}$ & No \\
3 & $4(2+\sqrt{3})$ & No \\
7 & $4(8+3 \sqrt{7})$ & No \\
9 & $4\left(7+4 \sqrt[4]{12}+2 \sqrt[4]{12^{2}}+\sqrt[4]{12^{3}}\right)$ & No \\
15 & $4(28+16 \sqrt{3}+12 \sqrt{5}+7 \sqrt{15})$ & No \\
\hline
\end{tabular}

Proof. If $m \in \mathbb{N}$, then we can use the definition of $F_{(1,2)}(x)$ to show that

$$
F_{(1,2)}(\sqrt{m})=\sum_{n=1}^{\infty} \frac{b_{n}}{n^{2}},
$$

where $b_{n}$ has the stated generating function. Furthermore, equation (4.15) reduces to

$$
F_{(1,2)}(\sqrt{m})=\frac{\pi^{2}}{16 \sqrt{m}} m\left(8 i g_{m}^{8} G_{m}^{4}\right) .
$$


Therefore, we can obtain Mahler measure formulas by appealing to tables of class invariants [5]. In order to check the values of $8 g_{m}^{8} G_{m}^{4}$, we can solve (7.1) to show that

$$
\begin{aligned}
8 g_{m}^{8} G_{m}^{4} & =4\left(G_{m}^{12}+\sqrt{G_{m}^{24}-1}\right) \\
& =4 \sqrt{2} g_{m}^{6} \sqrt{g_{m}^{12}+\sqrt{g_{m}^{24}+1}}
\end{aligned}
$$

For example, since $G_{1}=1$, it follows that $8 g_{1}^{8} G_{1}^{4}=4$. While this type of argument frequently leads to nested radicals, many of those identities simplify with sufficient effort.

A cursory inspection of Theorem [7.1, reveals only one instance where $b_{n}$ is multiplicative. In particular, when $m=1$ the generating function for $b_{n}$ reduces to $e_{4}^{2} e_{8}^{2}$. This cusp form is associated to a conductor 36 elliptic curve with complex multiplication [20]. Thus, we have given a new proof of the formula for $m(4 i)$ [22]. When $m=2$, a brief computation reveals that $b_{3}=b_{11}=0$, but $b_{33}=-8$, and therefore $e_{8}^{5} / e_{16}$ is not multiplicative. Fortunately, Somos has pointed out that $e_{8}^{5} / e_{16}$ is the real part of a multiplicative cusp form in $\mathbb{Q}(i \sqrt{2})$ :

$$
\begin{aligned}
g(q) & =\frac{e_{8}^{5}}{e_{16}}+2 i \sqrt{2} \frac{e_{16}^{5}}{e_{8}} \\
& =q+2 i \sqrt{2} q^{3}-5 q^{9}+\ldots
\end{aligned}
$$

It is not difficult to find a formula for $L\left(e_{16}^{5} e_{8}^{-1}, 2\right)$, and therefore we can show that

$$
\frac{16 \sqrt{2}}{\pi^{2}} L(g, 2)=m(4 i \sqrt{2 \sqrt{2}+2})+\frac{i}{\sqrt{2}} m(4 \sqrt{2 \sqrt{2}-2}) .
$$

It would probably be interesting to determine if $g(q)$ holds some special arithmetic significance.

\section{Higher polylogarithms and conclusion}

We will conclude the paper, by showing that the method from Section 4 can be used to produce identities for elliptic polylogarithms. Let us briefly reexamine Proposition 4.4. If we had used involutions for weight $1 / 2$ theta functions, rather than involutions for weight $3 / 2$ theta functions, we would have obtained formulas including

$$
\frac{F(1,1,1, x)}{(3+x)^{2}}=\frac{\pi}{36 \sqrt{x}} \sum_{n=1}^{\infty} \chi(n) D\left(i e^{-\frac{\pi n}{\sqrt{12 x}}}\right),
$$

where $D(z)=\operatorname{Im}\left(\operatorname{Li}_{2}(z)+\log |z| \log (1-z)\right)$ is the Bloch-Wigner dilogarithm, and $\chi(n)$ is a character modulo 12 , with $\chi(1)=\chi(11)=1$, and $\chi(5)=\chi(7)=-1$. It is also interesting to note that the following conjecture of Rodriguez-Villegas [12]:

$$
\mathrm{m}\left(1+x_{1}+x_{2}+x_{3}+x_{4}\right) \stackrel{?}{=} \frac{675 \sqrt{15}}{16 \pi^{5}} L(f, 4),
$$


where $f(q)=e_{3}^{3} e_{5}^{3}+e_{1}^{3} e_{15}^{3}$, can be reformulated using such an argument. By the method of Proposition 4.4, we have

$$
L(f, 4)=-\frac{128 \pi}{15^{3}} \sum_{n=1}^{\infty} \chi_{-4}(n)\left(9 R\left(i q^{n}\right)+R\left(i q^{3 n}\right)\right)
$$

where $R(z)=\operatorname{Im}\left(\log |z| \operatorname{Li}_{4}(z)-\log ^{2}|z| \operatorname{Li}_{3}(z)+\frac{\log ^{3}|z|}{3} \operatorname{Li}_{2}(z)\right)$, and $q=e^{-\pi \sqrt{15} / 6}$.

\section{Acknowledgements}

The author would like to thank David Boyd, Wadim Zudilin, Larry Glasser, Bruce Berndt, Michael Somos, and Anton Mellit for their useful comments and encouragement. Additionally, the author thanks the Max Planck Institute of Mathematics for their hospitality.

\section{References}

[1] V. S. Adamchik, A certain series associated with Catalan's constant. Z. Anal. Anwendungen 21 (2002), no. 3, 817-826.

[2] G. E. Andrews, R. Askey, R. Roy, Special functions. Encyclopedia of Mathematics and its Applications, 71. Cambridge University Press, Cambridge, 1999.

[3] G. E. Andrews and B. C. Berndt, Ramanujan's Lost Notebook, Part I, Springer-Verlag, New York, 2005.

[4] B. C. Berndt, Ramanujan's Notebooks, Part III, Springer-Verlag, New York, 1991.

[5] B. C. Berndt, Ramanujan's Notebooks, Part V, Springer-Verlag, New York, 1998.

[6] D. Borwein, J. M. Borwein and K. F. Taylor, Convergence of lattice sums and Madelung's constant. J. Math. Phys. 26 (1985), no. 11, 2999-3009.

[7] D. W. Boyd, Mahler's measure and special values of L-functions, Experiment. Math. 7 (1998), 37-82. Academic Press, 1994.

[8] F. Brunault, explicite du thorme de Beilinson pour la courbe modulaire $X_{1}(N)$. C. R. Math. Acad. Sci. Paris 343 (2006), no. 8, 505-510.

[9] L. Carlitz, Note on some partition formulae. Quart. J. Math., Oxford Ser. (2) 4, (1953). 168-172.

[10] R. E. Crandall, New representations for the Madelung constant. Experiment. Math. 8 (1999), no. 4, 367-379.

[11] C. Deninger, Deligne periods of mixed motives, $K$-theory and the entropy of certain $Z^{n}$-actions, J. Amer. Math. Soc. 10 (1997), no. 2, 259-281.

[12] S. Finch, Modular forms on $S L_{2}(\mathbb{Z})$, preprint, (2005). 
[13] S. Finch, Dedekind Eta Products, unpublished note (2007).

[14] P. J. Forrester and M. L. Glasser, Some new lattice sums including an exact result for the electrostatic potential within the NaCl lattice. J. Phys. A: Math. Gen. 15(1982), 911-914.

[15] M. L. Glasser, Evaluation of lattice sums. IV. A five-dimensional sum. J. Math. Phys. 16 (1975), 1237-1238.

[16] N. Kurokawa and H. Ochiai, Mahler measures via crystalization, Commentarii Mathematici Universitatis Sancti Pauli, 54 (2005), 121-137.

[17] M. N. Lalín and M. D. Rogers, Functional equations for Mahler measures of genus-one curves, Algebra and Number Theory, 1 (2007), no. 1, 87-117.

[18] M. N. Lalín, On a conjecture of Boyd, preprint (2009).

[19] N. N. Lebedev and R. A. Silverman, Special functions \& their applications, Dover Publications Inc., New York, 1972.

[20] Y. Martin and K. Ono, Eta-Quotients and Elliptic Curves, Proc. Amer. Math Soc. (125) 1997, no. 11, 3169-3176.

[21] A. Mellit, Elliptic dilogarithms and parallel lines, Preprint (2009).

[22] F. Rodriguez-Villegas, Modular Mahler measures I, Topics in number theory (University Park, PA, 1997), 17-48, Math. Appl., 467, Kluwer Acad. Publ., Dordrecht, 1999.

[23] M. D. Rogers, New ${ }_{5} F_{4}$ hypergeometric transformations, three-variable Mahler measures, and formulas for $1 / \pi$. Ramanujan J. 18 (2009), no. 3, 327-340.

[24] M. D. Rogers and B. Yuttanan, Somos's modular equations and lattice sums, preprint, (2009).

[25] I. J. Zucker, Madelung constants and lattice sums for hexagonal crystals. J. Phys. A 24 (1991), no. 4, 873-879. 